\title{
TÉCNICAS DE INSEMINAÇÃO ARTIFICIAL E USO DE DIFERENTES DOSES INSEMINANTES EM SUÍNOS
}

\author{
MOREIRA, Fabiana ${ }^{1}$ \\ FERREIRA, Carlos Eduardo Ranquetat ${ }^{2}$ \\ PANZARDI, Andrea ${ }^{3}$ \\ CORCINI, Carine Dahl ${ }^{4}$

\begin{abstract}
${ }^{1}$ Médica Veterinária, Doutora, Programa de Pós-Graduação em Veterinária da UFPEL, ${ }^{2}$ Médico Veterinário,
\end{abstract} \\ Doutorando do Programa de Pós-Graduação em Veterinária da UFPEL, ${ }^{3}$ Médica Veterinária, Doutora, TOPIGS \\ do Brasil, ${ }^{4}$ Médica Veterinária, Professora Doutora da Faculdade de Veterinária da UFPEL.
}

\section{RESUMO}

\begin{abstract}
A redução do número de espermatozoides por dose inseminante (DI)/serviço, sem o comprometimento da taxa de parição e tamanho de leitegada, deve garantir a manutenção e a sustentabilidade da alta capacidade produtiva em granjas comerciais. Inovações ao longo dos anos na tecnologia de inseminação artificial (IA), incluindo a IA intracervical (IAIC) e IA pós-cervical permitiram 0 uso de menor número de espermatozoides/dose. Algumas centrais de IA produzem DI heterospérmicas formadas pelo pool ou mistura de sêmen proveniente de dois ou mais machos. Porém, este tipo de DI pode mascarar ou compensar possíveis reduções da capacidade fecundante individual. Esta revisão tem como objetivo descrever as técnicas de IA mais utilizadas para a espécie suína e abordar o impacto da utilização comercial de doses homospérmicas e heterospérmicas. 0 método de IAIC é o mais utilizado em granjas tecnificadas, sendo a concentração utilizada padronizada de 3,0 a 3,5 x 109 espermatozoides/DI depositados no interior da cérvix. Em contrapartida na IA pós-cervical ou intrauterina (IAIU) o sêmen é depositado diretamente no corpo do útero, visando a redução do número de espermatozoides por DI (1 x109). A comprovação da fertilidade individual dos machos doadores de sêmen é um fator determinante quando se quer reduzir o número de espermatozoides e ao utilizar dose do tipo homospérmica com o uso de IAIC. Tecnologias de IA que permitam reduzir o número de espermatozoides e a utilização de doses homospérmicas que auxiliem na identificação de machos com alta fertilidade e geneticamente superiores podem contribuir para o aumento da eficiência produtiva dos planteis.
\end{abstract}

Palavras-chave: Reprodutores. Cérvix. Doses inseminantes. Sêmen. Útero. 


\section{INTRODUÇÃO}

A Inseminação Artificial (IA) é uma biotécnica que pode contribuir significativamente para o aumento da produtividade, possibilitando melhoria dos índices reprodutivos, bem como oferecer novas perspectivas tecnológicas à atividade (BORTOLOZZO et al., 2008a). Esta técnica se difundiu de forma crescente, principalmente, em virtude do surgimento de linhas genéticas de machos terminadores que agregaram às carcaças de sua progênie características desejáveis exigidas pela tipificação na indústria de carnes (BORTOLOZZO et al., 2005).

Uma estimativa da ABIPECS/ABCS demonstra que o rebanho nacional de fêmeas se manteve estável nos últimos seis anos, e em 2010 o plantel foi de 2,4 milhões de matrizes. Deste total, aproximadamente $50 \%$ das matrizes estão alojadas em granjas tecnificadas, representando em torno de 1,2 a 1,3 milhões de fêmeas (ABIPECS/ABCS, 2011).

Segundo Belstra (2002), grande parte das pesquisas em relação à técnica de IA tem como principal objetivo a redução do número de espermatozoides por DI/serviço, sem que haja comprometimento da taxa de parição e do tamanho de leitegada. Várias inovações na tecnologia de inseminação, tais como a IA pós-cervical (IA intrauterina e intrauterina profunda) são favoráveis ao uso do menor número de espermatozoides por inseminação (FOXCROFT et al., 2008).

O método tradicionalmente empregado para IA em suínos é a inseminação artificial intracervical (IAIC), a qual preconiza a utilização de uma pipeta que mimetiza o pênis do cachaço, permitindo assim a deposição do sêmen no canal cervical (WATSON; BEHAN, 2002). Na inseminação artificial intrauterina (IAIU) o sêmen é depositado diretamente no corpo do útero, tendo como objetivo principal a redução do número de espermatozoides por DI, além de ser considerado um método mais fisiológico que a deposição na IAIC, o qual ocorre na porção caudal do útero (PELTONIEMI et al., 2009).

Uma vez que a produção de ejaculados de alta qualidade permite a utilização de um número relativamente menor de machos, a seleção destes é bastante intensa, visando a utilização de reprodutores que apresentem melhor potencial reprodutivo (ROBINSON; BUHR, 2005). Além disso, a utilização da IA em um maior número de fêmeas a partir de um macho, possibilita 
maior avanço no melhoramento genético destes animais, tendo em vista que um reprodutor de alto potencial genético disseminará suas características com maior amplitude para sua progênie (VERBERCKMOES et al., 2004).

Algumas centrais de IA produzem doses inseminantes heterospérmicas formadas por pool ou mistura de sêmen proveniente de dois ou mais machos (DIZIUK, 1996). Porém, este tipo de dose inseminante com aproximadamente $3 \times 10^{9}$ espermatozoides pode mascarar ou compensar, possíveis reduções na fertilidade entre os indivíduos (WABERSKI et al., 2008). A utilização de reprodutores subférteis e ejaculados de baixa qualidade diminui a eficiência reprodutiva e, consequentemente, a margem de lucro do produtor. No entanto, a necessidade de melhorar continuamente a eficiência da produção de carne suína, sugere que a prática comercial de IA deverá envolver maior uso de reprodutores com elevado mérito genético, a fim de permitir a disseminação de características importantes de produção (FOXCROFT et al., 2008).

Esta revisão tem como objetivo descrever as técnicas de IA utilizadas para a espécie suína, além de abordar o impacto da utilização comercial de doses homospérmicas e heterospérmicas.

\section{INSEMINAÇÃO ARTIFICIAL (IA)}

O primeiro relato do uso da técnica de IA em suínos foi no Japão e Rússia, por volta da década de 30 (WENTZ; BORTOLOZZO, 1998), no entanto esta biotécnica foi introduzida no Brasil somente em meados da década de 70. A ampla difusão mundial da IA está relacionada a um aumento na comercialização de sêmen pelas centrais de IA (sistemas abertos) e também pelo aumento no número de programas de produção de sêmen em ambiente de granja, nos chamados sistemas fechados principalmente, com a redução do custo final de produção obtido com a implementação desta técnica, quando comparada à monta natural (MN) (BORTOLOZZO; WENTZ, 1997). Porém, ainda é possível observar granjas menos tecnificadas utilizando a técnica de $\mathrm{MN}$, que requer uma maior quantidade de machos no plantel, em função da reduzida relação macho:fêmea (M:F), em torno de 1:25 (CORRÊA et al., 2001). 
Em sistemas abertos as DI são produzidas e comercializadas por uma central de IA, neste caso, as doses são enviadas à propriedade e o produtor ou o funcionário realizam a IA. Este tipo de sistema está caindo em desuso pelo alto risco sanitário de disseminação de doenças, além do curto período de armazenamento das doses que podem perder sua viabilidade em casos de problemas de logística e transporte destas doses entre centrais e granjas (WENTZ et al., 2005). Em programas fechados as doses são produzidas por uma central de IA instalada na própria granja, neste caso a coleta, diluição, conservação e aplicação são realizadas na granja, por isso há a necessidade de infraestrutura mínima de laboratório e mão de obra tecnificada (TONIOLLI, 2002; WENTZ et al., 2005). Atualmente os programas abertos de IA são os mais utilizados no Brasil

A concentração espermática dos atuais programas de IAIC geralmente usam de 2,5 a $4 \times 10^{9}$ de espermatozoides por DI com volume total entre 70 e $100 \mathrm{ml}$ sendo realizada de duas a três IA durante o período estral. Estas condições limitam o número de doses que podem ser preparadas a partir de um ejaculado para aproximadamente 20-25 doses (MARTINEZ et al., 2005).

Nos últimos anos a IA em suínos teve grande evolução, tanto como um meio de aumentar a produtividade (maior taxa de parição e maior número de leitões nascidos) devido a eliminação de ejaculados impróprios para o uso e pela maior segurança sanitária (WENTZ, et al., 2005), assim como melhorar a qualidade genética nos plantéis através da disseminação acelerada de material genético de animais superiores (CORRÊA et al., 2001). O número de espermatozoides por DI diminuiu continuamente de 6 × 109 para 3 × 109 quando utilizada em IAIC. Essa redução melhorou significativamente a eficiência da utilização dos machos doadores (WABERSKI et al., 2008). No entanto com o uso a IA, algumas limitações devem ser consideradas para a obtenção de índices satisfatórios, como: a qualificação das pessoas envolvidas no processo; o emprego de manejo reprodutivo adequado à técnica de IA; a necessidade da proximidade a uma central de IA ou sua implantação na própria granja, devido ao curto período de conservação do sêmen (VIANA, 1998). 


\section{MÉTODOS DE IA}

\section{Inseminação artificial intracervical (IAIC)}

O método de IAIC é o mais utilizado em granjas tecnificadas. Com a obtenção de um maior número de DI por macho quando comparada à MN é possível atingir com boas condições de manejo, uma produção anual de até 2.000 DI/ano/macho (BENNEMANN et al., 2007). De acordo com Corrêa et al., (2001) é possível verificar que em sistemas que utilizavam MN e passaram para a técnica de IAIC, a relação macho:fêmea que era em média de 1:18, passa a ser de 1:80 à 1:200, respectivamente. 0 uso da IA é justificado, pois o número de machos necessários no plantel é inferior ao requerido com a utilização da $\mathrm{MN}$, por exemplo, em uma granja de 500 matrizes seriam necessários 22 a 25 machos em regime de $\mathrm{MN}$, apenas cinco seriam necessários quando utilizada a IA.

Atualmente na maioria das centrais de IA são confeccionadas DI entre três a quatro bilhões de espermatozoides (BENNEMANN, 2008; HERNÁNDEZ-CARAVACA et al., 2012). Sendo DI geralmente utilizadas na IAIC em concentração espermática de 2,5-3,0 $\times 10^{9}$ espermatozoides/DI (CORRÊA et al., 2001; MARTINEZ et al., 2005). Esta concentração foi estipulada, tendo-se em vista que o sêmen é depositado no interior da cérvix, ficando grande parte do mesmo retido nas criptas cervicais, primeira barreira. Posteriormente, as células espermáticas migram até a junção útero-tubárica (JUT), que consiste na porção caudal do istmo, a qual atua como uma barreira para o transporte e reservatório dos espermatozoides (LANGENDIJK et al., 2005). Portanto, próximo ao momento de ovulação o restante das células devem percorrer um longo trajeto, desde a porção caudal da cérvix até a junção ampola-istmo, local onde ocorre a fecundação (RATH, 2002).

Além destas barreiras, outro inconveniente associado à IAIC é a ocorrência de refluxo durante, que ocorre tanto na MN quanto na IA. Segundo Dallanora et al. (2004), a ocorrência de maior ou menor refluxo durante a IAIC pode ser decorrente do volume da dose inseminante, impaciência e/ou inabilidade do inseminador durante o processo de IA e de variações na contratilidade uterina. Em suínos, a ocorrência de refluxo é um evento fisiológico, que leva a alterações nas taxas de fertilização, somente se a DI apresentar concentração igual ou inferior a $1 \times 10^{9}$ espermatozoides. Este efeito foi comprovado por 
Watson e Behan (2002), que observaram um baixo número de nascidos ao avaliar IA que utilizaram concentrações espermáticas de $1 \times 10^{9}$ quando comparado a DI de $3,2 \times 10^{9}$ espermatozoides/DI.

Estudo conduzido por Bennemann et al. (2007) demonstrou que fêmeas submetidas a IAIC com concentração de $3,0 \times 10^{9}$ espermatozoides/DI, e IAIU com DI contendo 0,5 $\times 10^{9}$, armazenadas por no máximo $48 \mathrm{~h}$ e com a IA (em intervalos de 24 horas) realizada no momento de ovulação, guiada por ultrassom, não apresentaram diferenças entre o total de embriões e a sobrevivência embrionária.

Formas de utilização da dose inseminante (DI):

- À fresco: imediatamente ou até 3 horas após a preparação das $\mathrm{DI}$, as mesmas poderão ser utilizadas (GORDON, 1999).

- Refrigerada à temperatura de 15 - 18 으: técnica mais difundida nas centrais de IA, com manutenção das $\mathrm{DI}$ em caixas acondicionadoras por no máximo $72 \mathrm{~h}$ em diluentes de curta duração, ou por períodos superiores a $72 \mathrm{~h}$ até $120 \mathrm{~h}$ em diluentes de longa duração (GERRITS et al., 2005). Estes cuidados são indispensáveis, uma vez que o espermatozoide suíno é particularmente sensível ao resfriamento quando exposto a temperaturas inferiores a $15^{\circ} \mathrm{C}$, diminuindo sua sobrevivência (BORTOLOZZO et al., 2008b). Fenômeno atribuído a alterações estruturais e bioquímicas que levam a ruptura da membrana plasmática, degeneração do acrossoma e perda da permeabilidade seletiva da membrana (BORTOLOZZO et al., 2008b). O acondicionamento deve promover a manutenção da qualidade espermática, de modo que a motilidade não deva ser menor que $60 \%$ antes da inseminação, mantendo mais de 2,5 bilhões de células vivas por dose (CORRÊA et al., 2001), assim como apresentar um volume final maior que $80 \mathrm{ml}$ e anormalidades espermáticas totais menor que $20 \%$ por DI (CBRA, 1998).

- Congelada: a técnica de criopreservação do sêmen suíno foi estabelecida por Pursel e Johnson (1975), porém não é aplicada como rotina, pelo fato do sêmen congelado apresentar, após o descongelamento, uma baixa taxa de fecundidade (cerca de 60 
\%), o que inviabiliza seu uso em granjas comerciais. Segundo Roca et al. (2003), o uso de sêmen congelado nos programas de IAIC, é ainda muito limitado, devido a baixas taxas de concepção em relação ao sêmen refrigerado (cerca de 20 \% a menos), apesar de ainda ser utilizado altas concentrações entre 5 e $6 \times 10^{9}$ de espermatozoides/DI, duas a três vezes por estro (ROCA et al., 2011). Entretanto, Roca et al. (2002), não observaram diferenças na taxa de concepção e no tamanho de leitegada utilizando IA intra-uterina (IAIU), comparando sêmen refrigerado e congelado. Estes achados indicam que a IAIU terá papel importante para viabilizar o uso de sêmen congelado em suínos em nível comercial (RATH, 2002).

\section{Inseminação Artificial Intra-uterina (IAIU)}

O primeiro relato da realização da técnica de IAIU em fêmeas suínas foi feito por Hancock em 1959 (apud BORTOLOZZO et al., 2005), utilizando DI de 0,1 x $10^{9}$ a $10 \times 10^{9}$ espermatozoides em volume de 20 e $120 \mathrm{ml}$. Este autor observou que a fertilidade das fêmeas foi melhor ao utilizarem a DI somente com $20 \mathrm{ml}$. Para a realização desta técnica, foram desenvolvidos cateteres flexíveis, de 3-5 mm de diâmetro, que foram introduzidos no interior da pipeta de IAIC ( $8 \mathrm{~mm}$ de diâmetro), a qual era previamente introduzida na cérvix, estendendo-se cerca de $20 \mathrm{~cm}$ além da pipeta convencional. Portanto, a DI é depositada no corpo do útero das fêmeas (WATSON; BEHAN, 2002). Em contrapartida, foram também desenvolvidos cateteres que não utilizam a pipeta de IAIC como guia. Watson e Behan (2002) verificaram que 95\% das fêmeas inseminadas através de IAIU não apresentaram nenhum tipo de resistência durante a introdução do cateter, sendo que as $5 \%$ restantes apresentaram uma leve resistência durante sua passagem através da cérvix. Portanto, é necessário realizar uma leve pressão para que o cateter atinja o útero, reduzindo a ocorrência de lesões.

A utilização da IAIU não é recomendada em leitoas nulíparas, com restrição em primíparas, pois estas categorias de fêmeas não apresentam seu trato reprodutivo totalmente desenvolvido, possuindo a cérvix mais estreita, o que pode ser associado com a ocorrência de lesões, culminando em descarte precoce das matrizes (DIEHL, et al., 2006). Além disso, a 
passagem do cateter utilizado neste tipo de IA provoca lesões e sangramento na cérvix e corpo uterino, levando ao aparecimento de retornos ao estro, principalmente em nulíparas (dados não publicados). Com a tecnologia disponível, ainda não é possível empregar a IAIU em leitoas, porém em fêmeas primíparas os resultados são bastante promissores (BORTOLOZZO, et al. 2005b), quando utilizado para a passagem da cérvix o cateter com auxílio da pipeta tipo Melrose (DIEHL, et al., 2005).

Serret et al. (2005), utilizaram fêmeas primíparas e observaram efeitos negativos sobre o tamanho total da leitegada com IAIU nas concentrações de 2,0, 1,0 e 0,5 x10 espermatozoides/DI, e também da interação entre ordem de parto e concentração espermática sobre a taxa de parição, além de efeitos negativos sobre o desempenho das fêmeas primíparas, quando comparadas com a IAIC.

Na IAIU, há um menor número de barreiras mecânicas e fisiológicas (região útero-tubárica e transporte sustentado) a serem vencidas pelos espermatozoides durante seu trajeto, até o local de fecundação (WATSON; BEHAN, 2002). Desta forma, a duração do trajeto a ser percorrido até o oviduto se torna menor, o que pode se refletir na obtenção de índices satisfatórios de desempenho reprodutivo com menores concentrações espermáticas (DALLANORA et al., 2004). A deposição do sêmen no terço inicial/médio do útero facilita sua rápida progressão, permitindo maior retenção dos espermatozoides no trato genital, mesmo quando observado um aumento no refluxo em relação ao volume e número de espermatozoides (DALLANORA et al., 2004). Segundo os autores, não foram observadas diferenças na proporção de espermatozoides refluídos nas doses de $1,0 \times 10^{9}$ espermatozoides e de 0,25 × $10^{9}$ espermatozoides, mas a quantidade de sêmen que permaneceu no trato reprodutivo da fêmea foi quatro vezes superior com a dose mais elevada.

Watson e Behan (2002) compararam na técnica de IAIU, três diferentes concentrações (3,0, 2,0 e 1,0 × $10^{9}$ espermatozoides/DI), e não observaram diferenças em taxas de parição e total de leitões nascidos entre estas concentrações. Entretanto, DI com concentração de $0,5 x$ $10^{9}$ espermatozoides podem apresentar resultados variáveis em que foram encontradas taxas de parição e tamanho de leitegada reduzidos com DI na referida concentração 
(DALLANORA et al., 2004; SERRET et al., 2005). Em DI de 1,5 x 109 espermatozoides/40ml, ao utilizar a técnica de IAIU os parâmetros de fertilidade, tais como taxa de parição, número de leitões nascidos e número de leitões nascidos vivos, foram significativamente superiores comparado ao uso da técnica tradicional de inseminação a IAIC com DI de 3,0 x 109 espermatozoides/80 $\mathrm{ml}$ em porcas com ordem de parto 2-3 (HERNÁNDEZ-CARAVACA et al., 2012). Ainda neste estudo as taxas de retorno ao estro foram reduzidas quando a técnica aplicada foi a IAIU na referida dose e volume de sêmen em relação a IAIC, ou seja, $4.57 \pm$ $0.20 \%$ e $6.47 \pm 0.24 \%$ respectivamente $(p<0.05)$.

Portanto, baseado neste contexto é possível confirmar que a IAIU com 1,0 e 1,5 x $10^{9}$ espermatozoides/DI é uma técnica simples, efetiva, segura de se realizar e que permite alcançar resultados semelhantes, quando comparado à 3,0 e 2,0 × $10^{9}$ na IAIC, comumente utilizados na IAIC não afetando os parâmetros de fertilidade (VAZQUEZ et al., 2008).

Dallanora et al. (2004), utilizaram $3 \times 10^{9}$ espermatozoides/90 $\mathrm{ml}$ na IAIC e 1,5 x $10^{9}$ espermatozoides/60 $\mathrm{ml}$ na IAIU não registraram diferenças nas taxas de concepção e parição, bem como no tamanho total de leitegada. Já em contrapartida Panzardi et al. (2010) observaram diferenças ao realizarem IAIC e IAIU nas taxas de parição, sendo de 94,7 \% e $85,3 \%$ respectivamente, e nas respectivas doses de $3,0 \times 10^{9} \mathrm{sptz} / 85 \mathrm{ml}$ e $1,5 \times 10^{9} \mathrm{sptz} / 60 \mathrm{ml}$. Entretanto não houve diferença no número de leitões nascidos totais, sendo de $13,7 \pm 0,3$ para a IAIC e 13,0 $\pm 0,3$ para a IAIU.

Em virtude da possibilidade de diminuição da DI (concentração espermática e volume) utilizada na técnica de IAIU, os reprodutores suínos passariam a ter um papel ainda mais importante no desempenho reprodutivo do plantel, pois serviriam a um número maior de matrizes. Assim, estes machos deveriam ser selecionados através de métodos mais seletivos para a estimativa de seu potencial fecundante, como por exemplo, baseando-se em técnicas in vitro (MACEDO et al., 2006; VERBERCKMOES et al., 2004).

A seleção de reprodutores para centrais de IA, em geral, é baseada na capacidade do seu potencial genético em transmitir à progênie características de desempenho de interesse econômico (ROBINSON; BUHR, 2005). Entretanto, mesmo com ótimo desempenho reprodutivo, estes reprodutores apresentam diferenças em desempenho de natureza 
individual, que se refletem nas taxas de fecundação (MEZALIRA et al., 2003). Estes autores, ao utilizarem 4 reprodutores de alto mérito genético em IAIU com concentrações espermáticas distintas (1,0, 0,5 e 0,25 x 10 ${ }^{9}$ espermatozoides/DI), observaram que em todas as concentrações, as taxas de prenhez superiores foram obtidas sempre com um mesmo macho, enquanto com a utilização de outro macho observou-se uma sensível redução na taxa de prenhez, quando a concentração foi de $0,25 \times 10^{9}$ espermatozoides/DI. Estes achados foram confirmados em outro trabalho que utilizou métodos distintos, tais como estimativa da qualidade seminal (motilidade progressiva, morfologia de cabeça, cauda e integridade de acrossoma) e da taxa de penetração in vitro na estimativa do potencial fecundante de reprodutores suínos (POPWELL; FLOWERS, 2004).

Os resultados de desempenho reprodutivo obtidos com a IAIU mostram que essa biotécnica possui aplicação prática e constitui uma ferramenta estratégica (BENNEMANN, 2008) devido a redução da DI com sucesso em porcas e ao mesmo tempo reduz custos (HERNÁNDEZCARAVACA et al., 2012).

\section{Inseminação Artificial Intra-uterina Profunda (IAIUP)}

Um procedimento diferente de inseminação intrauterina está sendo utilizado, o qual permite a deposição da dose de sêmen profundamente na porção caudal dos cornos uterinos, denominada de inseminação artificial intrauterina profunda (IAIUP) (WATSON; BEHAN, 2002). Com o uso desta técnica, é possível reduzir em vinte vezes o número de espermatozoides, e diminuir de oito a vinte vezes o volume da DI em relação a IA tradicional (IAIC) (MARTINEZ et al., 2005). Roca et al. (2003) demostraram que utilizando esta técnica foi possível a realização de inseminação artificial com doses de $150 \times 10^{6}$ espermatozoides com volume de $7,5 \mathrm{ml}$, reduzindo significativamente os custo com diluente por dose.

Em seu estudo Vazquez et al. (2005), demostrou que com doses de 50 e $100 \times 10^{6}$ espermatozoides em relação a $3 \times 10^{9}$ espermatozoides na IAIC a taxa de parição e tamanho de leitegada não diferiram entre os grupos, porém quando a técnica foi aplicada comercialmente, com DI de $150 \times 10^{6}$ espermatozoides, foi observado comprometimento nestes dois parâmetros de fertilidade. Entretanto, pesquisas complementares devem ser 
feitas a fim de definir qual seria o número mínimo de espermatozoides a serem utilizados por dose (BORTOLOZZO et al., 2005b).

Comparando-se ambas as técnicas de IA pós-cervical (IAIU x IAIUP), nas DI de 1,5 e 1,0 × $10^{9}$ espermatozoides, respectivamente, os parâmetros de fertilidade, como taxa de parição, número de leitões nascidos e nascidos vivos foram semelhantes $(p>0,01)$ em porcas de 2-5 partos, somente o número de leitões nascidos foi significativamente menor $(11,56 \pm 3,26)$ em fêmeas com ordem de parto $\geq 6$ quando a técnica utilizada foi a IAIUP, em relação a IAIU $(12,09 \pm 2,96)$ (HERNÁNDEZ-CARAVACA et al., 2012).

Uma das maiores dificuldades de se realizar esta técnica ocorre durante a introdução do cateter flexível através da cérvix e cornos uterinos de fêmeas nulíparas e primíparas, já que estas apresentam o canal cervical mais estreito que fêmeas multíparas (BORTOLOZZO et al., 2005a). Outra limitação é a maior necessidade de treinamento para tornar possível a implementação desta técnica em granjas comerciais, principalmente no que diz respeito à prática do inseminador em relação à introdução do cateter (MARTINEZ et al., 2004). Portanto, o emprego da IAIUP está relacionado com as inovações tecnológicas de uso com sêmen ou congelado ou sexado (VAZQUEZ et al., 2005).

\section{EFEITOS DA UTILIZAÇÃO DE DOSES HOMOSPÉRMICAS E HETEROSPÉRMICAS NA IA}

As técnicas de avaliação de sêmen usadas na maioria dos centros comerciais de IA, que incluem volume do ejaculado, motilidade progressiva, morfologia e concentração dos espermatozoides, fornecem uma estimativa muito conservadora da fertilidade individual de cada animal. Portanto, pequenas diferenças no potencial fecundante dos machos doadores são mascaradas pelo uso generalizado de sêmen heterospérmico para IA comerciais em muitos países (DYCK, 2011).

A redução do número de espermatozoides por DI pode evidenciar tanto machos com baixa fertilidade, como aqueles com fertilidade superior, que passariam despercebidos empregando-se doses com o número tradicional de espermatozoides (BORTOLOZZO et al., 2008b). Para tal, métodos eficazes para predizer a fertilidade dos machos seriam essenciais 
para excluir animais subférteis e, assim, otimizar o uso de animais com comprovado potencial fecundante e geneticamente superiores com um menor número de espermatozoides por dose na IA (DYCK, 2011).

Assim, uma grande redução na concentração da DI utilizada, em machos com pequenas diferenças em fertilidade, podem acarretar na exacerbação destas diferenças, quando estes machos forem utilizados em IA homospérmica (MEZALIRA et al., 2003), especialmente porque a capacidade fecundante destes machos pode ser mascarada quando estimada por técnicas convencionais de avaliação da qualidade espermática (STAHLBERG et al., 1998).

Em estudo realizado por Alm et al. (2006) foi avaliada a fertilidade de 50 machos utilizados em 10773 inseminações homospérmicas em doses com $2 \times 10^{9}$ espermatozoides e 96 machos usados para compor DI com $3 \times 10^{9}$ espermatozoides em 34789 inseminações homospérmicas. De acordo com os resultados, os autores recomendam a utilização de $3 \mathrm{x}$ $10^{9}$ espermatozoides/DI de sêmen homospérmico. Observando que deficiências morfológicas podem ser compensadas pelo maior número de espermatozoides utilizados, e que doses com menos de $3 \times 10^{9}$ espermatozoides levam ao decréscimo das taxas de fertilidade e tamanho das leitegadas, o que pode ser observado na (Tabela 1). A comprovação da fertilidade individual dos machos doadores é um fator determinante ao se reduzir o número de espermatozoides e ao se utilizar doses homospérmicas em IAIC (ALM et al., 2006).

Tabela 1. Parâmetros de qualidade seminal e desempenho reprodutivo utilizando diferentes concentrações espermáticas por dose inseminante homospérmica (média \pm DP).

\begin{tabular}{llll}
\hline Variável & Grupo A & Grupo B & P \\
& $(\mathbf{2} \times \mathbf{1 0 9})$ & $\mathbf{( 3 \times 1 0 9 )}$ & \\
\hline Taxa de não retorno (\%) & $75,8 \pm 8,8$ & $84,0 \pm 3,7$ & $<0.001$ \\
Tamanho de leitegada fêmeas primíparas & $10,1 \pm 1,0$ & $10,7 \pm 0,6$ & $<0.001$ \\
Tamanho de leitegada fêmeas multíparas & $11,7 \pm 0,7$ & $12,1 \pm 0,6$ & $<0.001$ \\
Espermatozoides normais (\%) & $85,6 \pm 15,3$ & $85,2 \pm 13,1$ & \\
Defeitos maiores (\%) & $9,9 \pm 14,7$ & $5,5 \pm 6,1$ & \\
Defeitos menores (\%) & $4,4 \pm 4,5$ & $9,4 \pm 10,9$ & \\
\hline
\end{tabular}

Adaptado de Alm et al., 2006. 
Porém este número relativamente elevado de espermatozoides utilizados na prática comercial de IA (aproximadamente $3 \times 10^{9}$ espermatozoides / dose) compensam possíveis reduções na fecundidade, o que pode ser demonstrado quando são utilizados machos com qualidade seminal inferior. Com isto, reprodutores subférteis podem estar sendo utilizados na rotina para a produção de doses (FOXCROFT et al., 2008).

Em recente estudo preliminar sobre o desempenho reprodutivo, Foxcroft et al. (2010a) avaliaram dois machos Duroc em rotina normal de coleta com parâmetros de qualidade do sêmen considerados aceitáveis (motilidade superior a $80 \%$ de e defeitos nos espermatozoides $<15 \%$ ), ambos com histórico de boa fertilidade. Os reprodutores foram avaliados através do uso de seu sêmen em IA com $2 \times 10^{9}$ espermatozoides/DI em doses heterospérmicas (pool de sêmen) e homospérmicas. Os resultados de produtividade foram positivos ao inseminar as fêmeas tanto com doses heterospérmicas quanto com homospérmicas. No entanto foi observada uma diferença de 2,5 embriões totais no dia 30 de gestação, devido a uma diferença de $10 \%$ na taxa de fecundação e/ou de sobrevivência embrionária nesta etapa de prenhez (Tabela 2). O melhor desempenho do Macho A, foi mascarado quando o sêmen deste indivíduo animal foi misturado ao outro reprodutor (Macho B).

A adoção de uma estratégia de IA com dose homospérmica pode melhorar os números totais de suínos nascidos, permitindo que o reprodutor expresse seu verdadeiro potencial reprodutivo (FOXCROFT et al., 2010a).

Tabela 2. Comparação de dois machos utilizados em protocolos de inseminação homospérmica e heterospérmica com dois bilhões de espermatozoides por dose.

\begin{tabular}{lccc}
\hline \multicolumn{1}{c}{ Variável } & $\begin{array}{c}\text { "Pool" doses } \\
\text { (heterospérmica) }\end{array}$ & $\begin{array}{c}\text { Macho A } \\
\text { (homospérmica) }\end{array}$ & $\begin{array}{c}\text { Macho B } \\
\text { (homospérmica) }\end{array}$ \\
\hline Fêmeas inseminadas & 32 & 11 & 14 \\
Taxa de ovulação* & 20,3 & 20,7 & 20,3 \\
Embriões vivos 30o dia de gestação* & 15,2 & 17,7 & 15 \\
Taxa de sobrevivência embrionária (\%)* & 75 & 85 & 75 \\
\hline
\end{tabular}

*média de valores

Fonte: Centro de Pesquisa e Tecnologia em Suínos da Universidade de Alberta, dados não publicados, 2009. 
Em outro estudo Foxcroft et al., (2010a), compararam proteínas do plasma seminal de dois machos suínos que apresentaram diferenças de fertilidade. O estudo destacou o problema de relacionar a progênie total nascida para cada macho doador quando utilizado o sêmen heterospérmico para a composição das Dls. Os dois machos estudados apresentaram parâmetros de qualidade de sêmen aceitáveis e semelhantes durante a coleta, testados em através de doses homospérmicas (30 inseminações por macho). Posteriormente o sêmen dos dois animais foi misturado ao sêmen de mais três animais sendo que o pool (cinco animais) foi formado por contribuição igual entre os doadores. Foram avaliados 104 leitões nascidos de doses heterospérmicas através de teste de paternidade, sendo identificados 31 animais como filhos do macho B e apenas um leitão filho do macho A. Estes dados destacam a importância de utilizar inseminações com doses homospérmicas aumentando a produtividade e reduzindo a utilização de machos de baixa fertilidade.

O impacto econômico da utilização de machos geneticamente superiores deve ser objetivado pela indústria produtora de carne suína, através do maior número de fêmeas inseminadas/reprodutor. Isto pode ser alcançado através da remoção de machos subférteis e da melhor utilização de reprodutores de alto potencial fecundante e geneticamente superiores, através das técnicas convencionais de avaliação da qualidade seminal e de seu uso em doses homospérmicas reduzindo o número de espermatozoides por DI (FOXCROFT et al., 2010b). O aumento da eficiência produtiva de carne suína deve envolver o uso intensivo de machos com alto desempenho reprodutivo e o mais alto mérito genético para características de produção (DYCK et al., 2011).

\section{CONCLUSÃO}

A biotécnica de IA está cada vez mais difundida entre as granjas comerciais de suínos, tanto com a prática de IAIC como IAIU. Estas biotecnologias visam a utilização de machos geneticamente superiores, redução de contaminantes, do número de machos dentro do plantel e a qualificação da mão de obra, tendo como consequência a otimização dos resultados de produtividade. 
Além disto, em virtude da grande competitividade da cadeia produtiva de suínos e das pequenas margens de lucro obtidas, fazem com que se lance mão de métodos que permitam aumentar e/ou melhorar o desempenho destas atividades. Neste contexto, tecnologias de IA que permitam reduzir o número de espermatozoides e a utilização de doses inseminantes homospérmicas que auxiliem na identificação de machos com alta fertilidade e geneticamente superiores, podem contribuir para o aumento da eficiência dentro da cadeia produtiva.

\section{ARTIFICIAL INSEMINATION TECHNIQUES AND DIFERENTS INSEMINATION DOSES IN SWINE}

\section{ABSTRACT}

$\mathrm{R}$ educing the number of sperm per insemination dose (ID) / service without compromising the farrowing rate and litter size, must ensure the maintenance and sustainability of high production capacity on commercial farms. Innovations over the years in the technology of artificial insemination (AI), including intra-cervical AI (IAIC) and IA post-cervical allowed the use of fewer sperm / dose. Some central of Al produce ID heterospermic formed by pooling or mixing of semen from two or more males. However, this type of ID can mask or compensate possible reductions in the fertilizing capacity. This review aims to describe the $\mathrm{Al}$ techniques most commonly used for swine and address the impact of the commercial use of doses homospermic and heterospermic. The method of IAIC is the most used in farms technicality, the concentration used standardized 3.0 to $3.5 \times 109$ sperm / $\mathrm{ml}$ disposed within the cervix. By contrast, in intrauterine Al (IAIU) semen is deposited directly into the body of the uterus in order to reduce the number of sperm per DI (1 x109). The proof of the fertility of individual semen donors is an important factor when one wants to reduce the number of sperm dose and the use of type homospermic using IAIC. Al technologies that allow to reduce the number of spermatozoids and the use of doses homospermic to assist in identifying male fertility and a high genetically superior may contribute to increase production efficiency.

Keywords: Boars. Cervix. Insemination dose. Semen. Uterus. 


\section{TECNICAS DE INSEMINACIÓN ARTIFICIAL Y EL USO DE DIFERENTES DOSIS DE INSEMINACIÓN EN CERDOS}

\section{RESUMEN}

a reducción del número de espermatozoides por dosis de inseminación (DI) / servicio sin comprometer la tasa de partos y tamaño de la camada, debe velar por el mantenimiento y la sostenibilidad de la capacidad de producción de alta en granjas comerciales. Las innovaciones en los últimos años en la tecnología de inseminación artificial (IA), como intra-cervical IA (IAIC) e IA post-cervical permite el uso de menos espermatozoides / dosis. Algunos centros de inseminación artificial producen DI heterospérmicas formadas por la agrupación o la mezcla de semen a partir de dos o más machos. Sin embargo, este tipo de DI puede enmascarar o compensar posibles reducciones en la capacidad del indivíduo para fertilizar. Esta revisión tiene como objetivo describir las técnicas de IA más utilizadas para los cerdos y abordar el impacto de la utilización comercial de las dosis heterospérmicas y homospérmicas. El método de inseminación artificial IAIC es el más utilizado en las granjas con tecnificación, siendo la concentración estándar utilizada 3,0 a 3,5 x $10^{9}$ espermatozoides / DI depositado dentro de la cervix. Por el contrario en IA cervical o intrauterino (IAIU), el semen se deposita directamente en el cuerpo del útero, con el fin de reducir el número de espermatozoides por DI $\left(1 \times 10^{9}\right)$. La prueba de la fertilidad de machos individuales donantes de semen es un factor importante cuando se quiere reducir el número de dosis de esperma y cuando se usa dosis homospérmicas en la IAIC. Las tecnologías de inseminación artificial para reducir el número de espermatozoides y la utilización de las dosis homospérmicas que ayudan en la identificación de los machos con alta fertilidad y superiores genéticamente pueden contribuir al aumento de la eficiencia de producción porcina.

Palabras clave: Porcinos. Cuello uterino. Inseminación dosis. Semen. Útero.

\section{REFERÊNCIAS}

ABIPECS/ABCS (2011) Relatórios anuais ABIPECS. Disponível em: http://www.abipecs.org.br/relatorios.html. Acesso em: 06 jun. 2012.

ALM, K.; PELTONIEMI, O. A. T.; KOSKINEN, E. et al. Porcine field fertility with two different insemination doses and the effect of sperm morphology. Reproduction Domestic. v. 41, p. 210-213, 2006.

BELSTRA, B. A. Review: Intrauterine (Transcervical) and fixed-time artificial insemination in swine. College of Agriculture \& Life Sciences. Annual Swine Report, 2002. 
BENNEMANN, P. E.; KOLLER, F. L.; BERNARDI, M. L. et al. Desempenho reprodutivo de fêmeas suínas submetidas à inseminação intra-uterina ou à tradicional. Ciência Rural, v.37, n.6, p. 1735-1739, nov./dez. 2007.

BENNEMANN, P. E. Protocolos emergenciais para programas de inseminação artificial em suínos. Acta Veterinarie Science, n. 1, v. 36, p. 27-32, 2008.

BORTOLOZZO, F. P.; WENTZ, I. Inseminação artificial em suínos no Brasil. Revista Brasileira de Reprodução Animal, v. 21, p. 13-15, 1997.

BORTOLOZZO, F. P.; WENTZ, I.; DALLANORA, D. Situação atual da inseminação artificial em suínos. Acta Scientiae Veterinarie, v. 33, n. 1, p. 17-32, 2005.

BORTOLOZZO, F. P.; BERNARDI, M. L.; BENNEMANN, P. E. et al. Inseminação Artificial em Suínos. In: GONÇALVES, P. B. D.; FIGUEIREDO, J. R.; FREITAS, V. J. F. Biotécnicas Aplicadas à Reprodução Animal. São Paulo: Roca, 2008a. Cap. 4, p. 125-144.

BORTOLOZZO, F.P., GOLDBERG, A. M. G.; WENTZ I. Até onde é possível reduzir o número de espermatozoides empregados na inseminação infracervical em suínos sem comprometer a fertilidade? Acta Scientiae Veterinariae, v. 36I, n. 1, p. 17-26, 2008 b.

CBRA. Manual para exame andrológico e avaliação de sêmen animal. Colégio Brasileiro de Reprodução Animal. 2. ed., Belo Horizonte, 1998. 49p.

CORRÊA, M. N.; MEINCKE, W.; LUCIA, T. JR.; DESCHAMPS, J. C. Inseminação Artificial em Suínos. Pelotas: Printpar, 2001, 188p.

DALLANORA, D.; MEZALIRA, A.; KATZER, L.H et al. Desempenho reprodutivo de fêmeas suínas inseminadas pela técnica intra-uterina ou tradicional. Pesquisa AgropecuáriaBrasileira, v. 39, n. 8, p. 815-819, 2004.

DYCK, M. K.; FOXCROFT, G. R.; NOVAK, S. et al. Biological Markers of boar fertility. Reproduction in Domestic Animals, v. 46, p. 55-58, 2011.

DIEHL, G. N.; AMARAL, W. S.; KUMMER, R. et al. Nova pipeta para inseminação intra-uterina em suínos. Ciência Rural, v. 36, n. 1, p. 179-185, jan./fev. 2006.

DZIUK, P. J. Factors that influence the proportion of offspring sired by a male following heterospermic insemination. Animal Reproduction Science, v. 43, p. 65-88, 1996.

FOXCROFT, G. R.; DYCK, M. K.; RUIZ-SANCHEZ, A. et al. Identifying useable semen. Theriogenology, v. 70, p. 1324-1336, 2008. 
FOXCROFT, G. R.; PATTERSON, J.; CAMERON, A.; et al. Application of advanced AI technologies to improve the competitiveness of the pork industry. Proceedings of the 21st IPVS Congress, Vancouver, p. 18-21, jul. 2010a.

FOXCROFT, G.; PATTERSON, J.; DYCK, M. Improving production efficiency in a competitive industry. In Manitoba Swine Seminar, Winnipeg, Canada, p. 81-98, $2010 \mathrm{~b}$.

GERRITS, R. J.; LUNNEY, J. K.; JOHNSON, L. A.; et al. Perspectives for artificial insemination and genomics to improve global swine populations. Theriogenology, v. 63, p. $283-299$, 2005.

GORDON, I. Inseminación artificial como método reproductor. En: Reproducción controlada del ganado vacuno y búfalos. Zaragoza: Acriba, 1999. Cap. 2, p. 41-57.

HERNÁNDEZ-CARAVACA, I.; IZQUIERDO-RICO, J. M.; MATÁS, C.; CARVAJAL, J. A.; VIEIRA, L.; ABRIL, D.; SORIANO-ÚBEDA, C.; GARCÍA-VÁZQUEZ, F. A. Reproductive performance and backflow study in cervical and post-cervical artificial insemination in sows. Animal Reproduction Science, v. 136, p. 14-22, 2012.

LANGENDIJK, P.; SOEDE, N. M.; KEMP, B. Uterine activity, sperm transport, and the role of boar stimuli around insemination in sows. Theriogenology, v. 63; p. $500-513,2005$.

MACEDO, M. C.; DESCHAMPS, J. C.; LUCIA, T. JR.; et al. In vitro penetration of fresh and vitrified swine oocytes by homologous spermatozoa using different incubation systems. Animal Reproduction Science, v. 92, p. 334-348, 2006.

MARTINEZ, E. A; CAAMAÑO, J. N; GIL, M. A et al. Successful nonsurgical deep uterine embryo transfer in pigs. Theriogenology, v. 61, p. 137-146, 2004.

MARTINEZ, E. A.; VAZQUEZ, J. M.; ROCA, J. et al. An update on reproductive technologies with potential short-term application in pig production. Reproduction Domestic Animals, v. 40, p. 300-309, 2005.

MEZALIRA, A.; DALLANORA, D.; SCHMIDT,A. C. T et al.Inseminação intrauterina com redução no volume e número de espermatozoides. In: XI Congresso Brasileiro de Veterinários especialistas em suínos. 2003. Goiânia. RESUMOS. Goiânia: Associação Brasileira de Veterinários Especialistas em Suínos, 2003, p. 217.

PANZARDI, A.; CORCINI, C. D.; MADEIRA, E. M.; PIASSI, L. M.; BESSEL, R. M. C.; PINTO, L. S.; DESCHAMPS, J. C.; LUCIA, T. JR. Genetic paternity test for piglets generated by heterospermic cervical and post-cervical artificial insemination. ActaVeterinariaBrasilica, v. 4, n. 3, p. 168-175, 2010.

PELTONIEMI, O. A. T.; ALM, K.; ANDERSON, M. Uterine insemination with a standard Al dose in a sow pool system. Reproduction Domestics Animals, v. 44, p. 414-418, 2009. 
POPWELL, J. M.; FLOWERS, W. L. Variability in relationships between semen quality and estimates of in vivo and in vitro fertility in boars. Animal Reproduction Science, v. 81, p. 97113, 2004.

RATH, D. Low dose insemination in the Sow - A Review. Reproduction of Domestic Animals, v. 37, p. 201-205, 2002.

ROBINSON, J. A. B.; BUHR, M. M. Impact of genetic selection on management of boar replacement. Theriogenology, v. 63, p. 668-678, 2005.

ROCA, J.; CARVAJAL, G.; CUELLO, C. et al. Fertility of cryopreserved boar spermatozoa after transcervical deep intrauterine insemination. Theriogenology, v. 57, p. 385, 2002.

ROCA, J.; CARVAJAL, G.; LUCAS, X. Fertility of weaned sows after deep intrauterine insemination with a reduced number of frozen-thawed spermatozoa. Theriogenology, v. 60, p. 77-87, 2003.

ROCA, J.; PARRILLA, I.; RODRIGUEZ-MARTINEZ, H.; GIL, M. A.; CUELLO, C.; VAZQUEZ, J. M.; MARTINEZ, E. A. Approaches towards efficient use of boar semen in the pig industry.

Reproduction in Domestic Animals, n. 2, v. 46, p. 79-83, 2011.

SERRET, C. G., ALVARENGA, M. V. F., CÓRIA, A. L. P. et al. Intrauterine artificial insemination in female swine with distinct sperm concentrations, parities and methods of ovulation estimation. Animal Reproduction, v. 2, p. 250-256, 2005.

STAHLBERG, R; HARLIZIUS, B.; WEITZE, K. F. et al. Identification of embryo paternity using polymorphic DNA markers to assess fertilizing capacity of spermatozoa after heterospermic insemination in boars. Theriogenology, v. 53, p. 1365-1373, 1998.

TONIOLLI, R. Aspectos de um programa de inseminação artificial em suínos. Ciência Animal, n. 1, v. 12, p. 7-17, 2002.

VAZQUEZ, J. M.; MARTÍNEZ, E. A.; ROCA, J.; GIL, M. A.; PARILLA, I.; CUELLO, C.; CARVAJAL, G.; LUCAS, X. VAZQUEZ, J. L. Improving the efficiency of sperm technologies in pigs: the value of deep intrauterine insemination. Theriogenology, v. 63, p. 536-547, 2005.

VAZQUEZ, J. M. J. ROCA, J.; M. A. GIL, C. CUELLO, I. PARRILLA, J. L. VAZQUEZ, E. A. MARTínEZ. New developments in low-dose insemination technology. Theriogenology, v. 70, p. 12161224, 2008.

VERBERCKMOES, S.; SOOM, A. V.; KRUIF, A. Intra-uterine insemination in Farm Animals and Humans. Reproduction in Domestic Animals, v. 39, p. 195-204, 2004.

VESSEUR, P. C.; KEMP, B.; HARTOG, L. A. Factors influencing the proportion if offspring from second insemination in sows. Animal Reproduction Science, v. 41, p. 255-265, 1996. 
VIANA, C. H. C. Relações entre as características intervalo desmame-cio, duração do cio e momento de ovulação pela ultra-sonografia e dosagem de progesterona sérica em fêmeas da espécie suína. São Paulo: USP, 1998. Dissertação (Mestre em Reprodução Animal). Faculdade de Medicina Veterinária e Zootecnia. Universidade de São Paulo, 1998.

WABERSKI, D.; PETRUNKINA, A. M.; TÖPFER-PETERSEN, E. Can external quality control improve pig Al efficiency? Theriogenology, v. 70, p. 1346-1351, 2008.

WATSON, P. F.; BEHAN, J. R. Intrauterine insemination of sows with reduced sperm numbers: results of a commercially based field trial. Theriogenology, v. 57, p. 1683-1693, 2002.

WENTZ, I.; BORTOLOZZO, F. P. Inseminação artificial em suínos. In: SOBESTIANSKY, J.; WENTZ, I.; SILVEIRA, P. R. S.; SESTI, L. A. C. Suinocultura intensiva: produção, manejo e saúde do rebanho. 2. ed. Concórdia: Embrapa-CNPSA, 1998. p. 209-220.

WENTZ, I.; BENNEMANN, P. E.; WOLLMANN, E. B.; BORTOLOZZO, F. P. Implantação de um programa de inseminação artificial em suínos. In: BORTOLOZZO, F. P.; WENTZ, I. et al. Inseminação artificial na suinocultura tecnificada. Porto Alegre: Pallotti, 2005. p.43-56.

Autor para correspondência: Fabiana Moreira - Programa de Pós-Graduação em Veterinária da UFPEL. Laboratório de Reprodução Animal, Campus Capão do Leão, Pelotas (RS). CEP 96010-900, CP 354. fabimorvet@gmail.com 